\title{
Síndrome de Weill-Marchesani
}

\author{
Dr. Jaime Herrera $V,{ }^{1}$; Dra. Margarita Morales N. ${ }^{2}$
}

Weill-Marchesani syndrome

\begin{abstract}
A patient with small stature, brachydactyly, broad metacarpals and phalanges, ventricular septal defect, smail spherical lens (spherophaquia) ectopia lentis (sub luxation) and glaucoma is presented. His mother shows similar, but attenuated signs. Both parents are cousins.

(Key words: short stature, brachydactyly, spherophaquia, Weill-Marchesani syndrome).
\end{abstract}

El síndrome de la braquidactilia-esferofaquia fue descrito originalmente por Weill en 1932 y fue asociado por este autor con el Sindrome de Marfan. En 1939 Marchesani separa ambos síndromes completando la descripción de Weill y clasificando estos cuadros como distrofias congénitas del mesodermo: Marchesani los divide en Formas hipoplásica (Marfan) e hiperplásica (W. Marchesani). Su frecuencia es bastante baja ya que sólo hay alrededor de 60 casos publicados en la literatura mundial. En Chile sólo hay 2 publicaciones ${ }^{1,3}$ con un total de 5 casos. Los signos más relevantes están dados por talla baja, cráneo ancho, órbitas pequeñas y poco profundas, hipoplasia maxilar, paladar estrecho, mala implantación dentaria, braquidactilia con metacar. pianos y falanges anchos y cortos, puede presentar cardiopatía: comunicación interventricular (CIV). En los ojos el cristalino es pequeño, esférico, con miopia lenticular y aún glaucoma. Un tercio de ellos puede llegar a la ceguera. Herencia: autosómica recesiva.

\section{Caso Clínico}

Niño de sexo masculino en quien se detectó un soplo cardíaco a la edad de 1 año, es referido a Genética a los 3 años 10 meses de edad por talla baja. Medía $94 \mathrm{~cm}$ de estatura (percentil 3) y pesaba $15 \mathrm{~kg}$ (percentil 25). Epicantus, mala implantación dentaria, braquidactilia, excesivo desarrollo muscular, limitación de la movilidad de los codos y de los dedos, obesidad, criptorquídea, soplo sistólico grado 3 ó 4 paraesternal izquierdo.

1. Departamento genética, Hospital San Juan de Dios.

2. Departamento of talmologia, Hospital San Juan de Dios.
Exámenes practicados: Electrocardiograma: desviación del eje eléctrico a derecha y signos de crecimiento bi ventricular. Radiografía de Tórax: aumento de cavidades derechas. Fonocardiograma compatible con C.I.V. En las radiografias del esqueleto se encontró: retraso de la edad ósea, acortamiento y engrosamiento de metacarpianos y falanges, clinodactilia, hipoplasia de la cabeza del radio. Otros exámenes como glicemia, nitrógeno ureico, $T_{\mathbf{a}}$, protrombina, hematocrito, cromatina sexual y cariograma fueron nomales. Los derma toglifos sólo presentaban discretas anomalías inespecificas, (ángulos atd $64^{\circ}$ y $58^{\circ}$, indice ab 47 y 50). El examen oftalmológico mostró: ectopia lentis, esferofaquia, miopía lenticular (Visión 0,33), cámara anterior estrecha (ecografía oftalmológica) y glaucoma, fondo de ojo normal. Urologia confirma criptorquidia con testículo derecho en ascensor e izquierdo pequeño atrófico.

El aiño debió ser operado en 1984 por \$s epiblefarón y en el mismo año por criptorquídea. El glaucoma se ha controlado con tratamiento medicamentoso y la miopía con lentes, conservando hasta ahora ambos nervios ópticos normales.

La madre presenta los mismos signos que el niño: talla baja, braquidactilia, tórax amplio, brazos cortos, cara redonda, pero ellos son menos intensos $y$ no tiene compromiso ocular (Figura 2). La genealogía es característica de herencia recesiva (Figura 3 ).

\section{COMENTARIO}

En este niño se nos plantea el diagnóstico diferencial de los sindromes de talla baja, bra. quidactilia y buen desarrollo muscular. Cada uno de ellos presenta signos característicos. El síndro- 


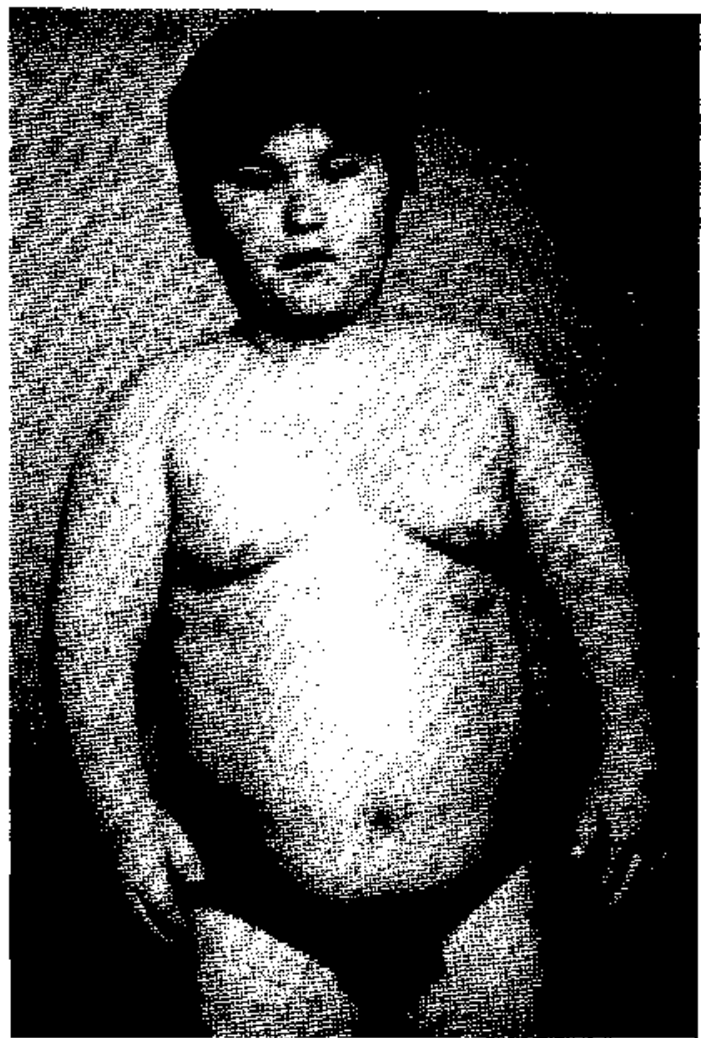

Figura 1. Síndrome de Weill-Marchevani: bracuidactilia, rigidez de las manos, ojos pequeños, corta estatura, tórtix ancho.

\section{GENEALOGIA WEILL MARCHESAN]}

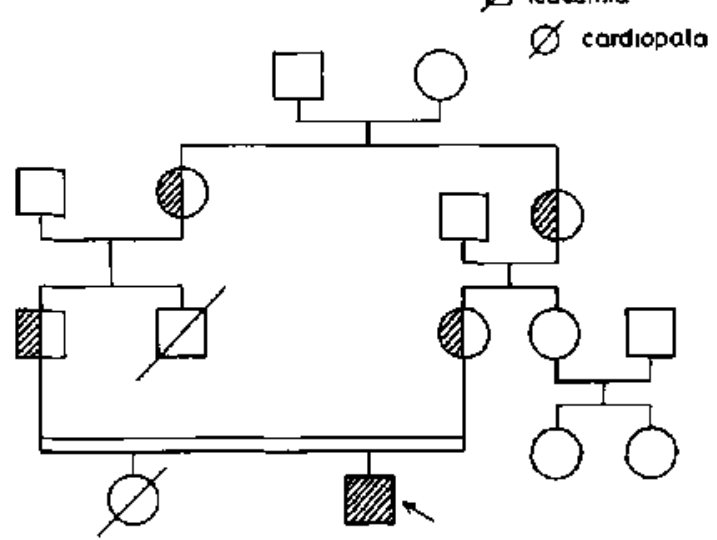

Medio achurado: portadores

Achurado completo: el probando

Figura 2. Genealogja típicamente recesirya en un paciente con sindronte de Weill-Marchesani. me (s) de Stanesco se caracteriza por braquicefalia. con calota gruesa acortamiento del segmento proximal); en el s. de Rubinstein-Taybi, los promeros ortejos y pulgares son anchos, grandes, mal implantados y la nariz es picuda; en el s. de Ccrnelia de Lange existe hipertricosis, sinofris, lazio con piquito central, oligodactilia y aún foromelia: en la braquuidactilia E los metacarpiano: $y$ metatarsianos son cortos y la cara tiene aspecto de luna llena; en la braquidactilia $A$ las falanges medias son hipoplásicas; la braquidactilia B se expresa con falanges terminales hipoplásicas; la hraquidactilia $\mathrm{C}$ muestra más de tres falanges por dedo; la braquidactilia $D$ tiene falanges terminales muy cortas; el s. de Turner es propio del sexo femenino; el s. de $\Lambda$ lbright muestra las clasicas alteraciones de calcio $y$ fósforo y la facie redonda. ${ }^{2.4-6}$

$\mathrm{Si}$ se agrega que el estudio de nuestro paciente most ró además de la braquidactilia la esferofa. quia y luxación de los cristalinos propios del Weill-Marchesani, no ofrece dudas su diagnós. tico y su caso resulta especialmente interasante, por su genealogia recesiva y las manifestaciones maternas en codo semejantes al niño, pero mucho methos intensas.

\section{RESUMEN}

Se presenta un paciente de baja estatura, braquidactilia, metacarpos y falanges anchos, defectos del tabique ventricular, esferofaquia, ectopia lentis y glaucoma. Su madre muestra signos similares pero atenuados. Ambos padres eran primos.

\section{REFERENCIAS}

1. Charlin, $C$ : Esferofaquia sub Juxación y braquidac tilia. Arch. Chil. Oftalmol. 11: 53, 1954.

2. Götz, M. Schenk, E.: Weill Merchasani syndrome, growth hormone, thyroid and chromosome studies. Z. Kinderheilt 116: 43, 1973.

3. Kuzmanic, A., Urutio, P.: Síndrome do Weill Merchasari: cuatro casos clínicos familiares. Arch. Chil. Oftalmol. 49: 29, 1983.

4. Smith, D.W.: Recognizable patterns of human malformation. Vol. VII 3t] Edition 1982, Saunders Company, Philadelphia, pág. 327.

5. Bergsma, D.: Birth defects compendium. 2nd Edition, 1979, National Foundation. March of Dimes New York, pág. 893.

6. Maroteaux, $P$.: Les maladies osseuses de l'enfant. 2nd Edition, 1982. Flamation Medicine Sciences, París, pág. 310 . 